\title{
Comparative Transcriptomics Atlases Reveals Different Gene Expression Pattern Related to Fusarium Wilt Disease Resistance and Susceptibility in Two Vernicia Species
}

\section{OPEN ACCESS}

Edited by:

Agnieszka Ludwików, Adam Mickiewicz University in Poznań, Poland

Reviewed by:

Sebastien Carpentier,

KU Leuven, Belgium

Taras P. Pasternak,

Albert Ludwigs University of Freiburg,

Germany

*Correspondence:

Yicun Chen

chenyc@caf.ac.cn

Yangdong Wang

wyd11111@126.com

${ }^{\dagger}$ These authors have contributed equally to this work.

Specialty section: This article was submitted to

Plant Biotechnology, a section of the journal Frontiers in Plant Science

Received: 03 October 2016 Accepted: 13 December 2016 Published: 27 December 2016

Citation:

Chen Y, Yin H, Gao M, Zhu H,

Zhang $Q$ and Wang Y (2016)

Comparative Transcriptomics Atlases

Reveals Different Gene Expression

Pattern Related to Fusarium Wilt

Disease Resistance and Susceptibility in Two Vernicia Species.

Front. Plant Sci. 7:1974.

doi: 10.3389/fpls.2016.01974

\author{
Yicun Chen ${ }^{* \dagger}$, Hengfu Yin ${ }^{\dagger}$, Ming Gao, Huiping Zhu, Qiyan Zhang and Yangdong Wang * \\ ${ }^{1}$ State Key Laboratory of Tree Genetics and Breeding, Chinese Academy of Forestry, Bejing, China, ${ }^{2}$ Institute of Subtropical \\ Forestry, Chinese Academy of Forestry, Hangzhou, China
}

Vernicia fordii (tung oil tree) is a promising industrial crop. Unfortunately, the devastating Fusarium wilt disease has caused its great losses, while its sister species (Vernicia montana) is remarkably resistant to this pathogen. However, the genetic mechanisms underlying this difference remain largely unknown. We here generated comparative transcriptomic atlases for different stages of Fusarium oxysporum infected Vernicia root. The transcriptomes of $V$. fordii and $V$. montana were assembled de novo and contained 258,430 and 245,240 non-redundant transcripts with N50 values of 1776 and 2452, respectively. A total of 44,310 pairs of putative one-to-one orthologous genes were identified in Vernicia species. Overall, the vast majority of orthologous genes shared a remarkably similar expression mode. The expression patterns of a small set of genes were further validated by quantitative real-time PCR. Moreover, 157 unigenes whose expression significantly correlated between the two species were defined, and gene set enrichment analysis indicated roles in increased defense response and in jasmonic and salicylic acid signaling responses during pathogen attack. Co-expression network analysis further identified the 17 hub unigenes, such as the serine/threonine protein kinase D6PK, leucine-rich repeat receptor-like kinase (LRR-RLK), and EREBP transcription factor, which play essential roles in plant pathogen resistance. Intriguingly, the expression of most hub genes differed significantly between $V$. montana and $V$. fordii. Based on our results, we propose a model to describe the major molecular reactions that underlie the defense responses of resistant $V$. montana to $F$. oxysporum. These data represent a crucial step toward breeding more pathogen-resistant $V$. fordii.

Keywords: Vernicia species, Fusarium wilt, comparative transcriptomics, resistance genes, co-expression

\section{INTRODUCTION}

Renewable biofuel has recently garnered much interest as a result of increased environmental awareness and the impact of fossil fuel-based energy. Vernicia fordii (Hemsl.) Airy-Shaw (Aleurites fordii Hemsl.) and Vernicia montana Lour. (A. Montana (Lour.) Wils.) belong to Euphobiaceae; this family includes many biomass-accumulating species, such as the physic nut (Jatropha curcas 
L.), castor bean (Ricinus communis L.), cassava (Manihot esculenta Crantz) and rubber tree (Hevea brasiliensis). Vernicia fordii and Vernicia montana are two Chinese native tung oil trees, and the oil that is refined from the seeds of Vernicia species has for centuries been used for fuel and industrial purposes. It was used as a fuel in ancient times and now is widely used in the production of paints, high-quality printing, plasticizers, and synthetic rubber. It finds particular use in special paints for the surfaces of weapons, steels, warships and submarines. Moreover, the oil from Vernicia species is an important raw material for biodiesel production (Brown and Keeler, 2005; Park et al., 2008; Chen et al., 2010; Shang et al., 2010). China collectively produces approximately 80,000 tons of tung oil per year, or approximately $70-80 \%$ of the world market (Zhan et al., 2012). However, this output of tung oil will not meet the needs of the international market in future years.

Vernicia fordii (tung oil tree or tung tree) and V. montana (wood oil tree) are the two main cultivars. Vernicia fordii outperforms $V$. montana in some agronomic traits, as it displays superior oil characteristics and faster maturation, while $V$. montana produces similar oil components and is resistant to wilt disease. Tung wilt disease, caused by the fungal pathogen Fusarium oxysporum Schlecht., is considered the most lethal disease of $V$. fordii and is a soil-borne fusarium disease. During infection, plants exhibit leaf chlorosis and slight vein clearing on outer leaflets, which is followed by leaf yellowing and abscission, discoloration of stem vascular tissue and death. To date, the disease cannot be managed unless the tree is grafted with $V$. montana (using $V$. montana as the parental stock), as the latter exhibits high resistance to F. oxysporum. They share similarity in morphology, anatomy and karyotype (chromosome number $2 \mathrm{n}=22=22 \mathrm{~m}$ ). Fusarium species are among the most important phytopathogenic and toxigenic fungi. Comparative genomics has revealed that the lineagespecific (LS) genomic region of $F$. oxysporum is related to pathogenicity and that LS transfer can alter strain pathogenicity (Ma et al., 2010). So far, more than 100 plant species have been attacked by $F$. oxysporum (Michielse and Rep, 2009). Recent studies on the molecular basis of F. oxysporum pathogenicity have been conducted with a limited number of hosts. The expression of the rice thaumatin-like protein gene in transgenic bananas enhances resistance to Fusarium wilt (Mahdavi et al., 2012). The tomato I-3 gene was also reported to confer resistance to Fusarium wilt disease (Catanzariti et al., 2015). Similarly, the transcription factor ERF72 conferred resistance to F. oxysporum (Chen et al., 2014b). However, little information is available on the genetic mechanism underlying the different resistance profiles of the two closely related Vernicia species.

In this study, we took advantage of the phylogenetic proximity between $V$. fordii and $V$. montana to compare the dynamic changes in gene expression during the process of $F$. oxysporum infection. We generated a comparative transcriptome between $V$. fordii and $V$. montana that consisted of four different $F$. oxysporum infection stages for each species.

\section{MATERIALS AND METHODS}

\section{Pathogen Isolation and Identification}

The pathogen was consistently isolated from the discolored vascular stem tissue of tung trees with wilt disease in Tianlin County, Guangxi Zhuang Autonomous Region, China, and it was then incubated on potato dextrose agar (PDA). Morphological features were first used to identify $F$. oxysporum. The fungus was further identified by PCR-based analysis of the ITS (internal transcribed spacer) and EF-1a (EF-1 $1 \alpha$ gene, translation elongation factor alpha) genes. Specific oligonucleotide primers were used for Fusarium identification (Supplemental Table S1) The PCR products were sequenced by Sangon Biotech Company, Shanghai.

Pathogenicity tests were conducted twice in a screen house. Four-week-old plants with wounded roots were submerged for 30 min in a conidial suspension $\left(1 \times 10^{6}\right.$ spores per $\mathrm{mL}$ diluted with sterile distilled water), while control plants were dipped in sterile distilled water. Symptoms observed on inoculated seedlings included leaf wilt (5 dpi, days post-inoculation), chlorosis, necrosis and plant death $(18 \mathrm{dpi})$. The pathogen was re-isolated from the stems of affected inoculated plants to confirm the cause of disease. The control plants did not exhibit symptoms. The fungus was identified by morphological features in combination with molecular identification (PCR of the ITS and EF-1a genes using the primers described above).

\section{Plant Growth and Pathogen Inoculation}

Vernicia fordii and Vernicia montana plantlets with two or three leaves and approximately $30 \mathrm{~cm}$ in height were used. All plantlets were kept under growth-chamber conditions at $26^{\circ} \mathrm{C}$ with a $16-\mathrm{h}$ light/8-h dark photoperiod. Before inoculation, each plantlet was sterilized in solutions containing $75 \%$ ethanol for $1 \mathrm{~min}, 0.5 \%$ sodium hypochlorite for $3 \mathrm{~min}$, and then $90 \%$ ethanol for $30 \mathrm{~s}$, followed by three rinses in sterile water.

A spore concentration of $1 \times 10^{6}$ spores per $\mathrm{mL}$, diluted with sterile distilled water, was used to inoculate the plants. Before inoculation, the plants were carefully removed from the soil, and the roots were rinsed with sterile water before being placed into the fungal inoculum for $30 \mathrm{~min}$. After inoculation, the plants grew under growth-chamber conditions at $26^{\circ} \mathrm{C}$, with relative humidity over $85 \%$ and a 16 -h light/ 8 -h dark photoperiod. The plants were then sampled at $2-15 \mathrm{dpi}$. The root, peel and leaves were cut and immediately frozen in liquid nitrogen and stored at $-80^{\circ} \mathrm{C}$ until retrieval for RNA preparation.

The three time points for harvesting the samples were selected as 2, 8, and 13 dpi according to the symptoms of the seedlings after infection.

\section{RNA Isolation, Preparation of cDNA Libraries and Illumina Sequencing}

Total RNA was extracted from the plant roots and from the rootstem transition region using the EASYspin plant RNA mini kit (Aidlab, Beijing, China). RNA integrity was confirmed using a UV-Vis Spectrophotometer Q5000 (San Jose, California, USA). All RNA samples displayed a 260/280 ratio of greater than 2.0 and RNA integrity numbers $(\mathrm{RIN}) \geq 8.0$. The mRNA was 
purified from $30 \mu \mathrm{g}$ of total RNA using Sera-mag Magnetic Oligo (dT) Beads (Illumina, Inc., San Diego, CA, USA) and was then fragmented into small pieces using divalent cations at an elevated temperature. The cDNA library was sequenced on the Illumina HiSeq ${ }^{\mathrm{TM}} 2000$ platform, and both ends of the inserts were sequenced. The 125-bp raw PE reads were generated by the Illumina Genome Analyzer II system (Illumina, Inc., San Diego, CA, USA).

For the RNA-Seq experiments, 4-week-old seedlings of resistant $V$. montana $(\mathrm{M})$ and susceptible $V$. fordii $(\mathrm{F})$ were each treated with F. oxysporum. According to the symptoms of the seedlings infected with the pathogen, 4 stages were defined for each species. The stages included untreated $V$. fordii (F0) and $V$. fordii infected with $F$. oxysporum at an early stage (F1), in the middle stage (F2) and at a late stage (F3); they also included untreated $V$. montana (M0) and $V$. montana infected with $F$. oxysporum at an early stage (M1), in the middle stage (M2) and at a late stage (M3). Three biological replicates were taken for each treatment. Therefore, in total, 24 RNA libraries were constructed and sequenced. RNA samples from the root and root-stem transition region were prepared for RNA-seq using Illumina HiSeq ${ }^{\mathrm{TM}} 2000$.

Equal amounts of RNA samples were then pooled for cDNA synthesis and RNA-seq. Before library construction, ribosomal RNA was removed; the cDNA libraries were constructed and sequenced by LC-Bio Co., Ltd, Hangzhou, China. Briefly, $10 \mu \mathrm{g}$ of total RNA for each group was used for library construction using the Truseq $^{\mathrm{TM}}$ RNA sample prep kit (Illumina, USA). The constructed DNA template was then enriched by PCR amplification (15 cycles). Amplicons were collected and purified by electrophoresis on gels made from Certified Low Range Ultra Agarose (Bio-Rad, USA). Before sequencing, the cDNA libraries were quantified using a TBS-380 micro fluorometer with the Picogreen reagent (Invitrogen, USA). Clone clusters were generated with an Illumina cBot using the Truseq PE Cluster Kit v3-cBot-HS, and high-throughput sequencing was performed on an Illumina Miseq sequencer using the Truseq SBS Kit v3-HS for 200 cycles.

\section{De novo Assembly of the Transcriptome}

By using Trinity 30, a de novo strategy was adopted to assemble the transcriptome of $V$. fordii and $V$. montana based on a total of 64.5 and 70 GB RNA-seq data (eight samples with three biological replicates for each). N50 number, average length, max length, and contig number during different length interval were all been calculated (Supplemental Table S2).

For the RNA-Seq experiments, 4-week-old seedlings of resistant $V$. montana (M) and susceptible $V$. fordii (F) were each treated with F. oxysporum. According to the symptoms of the seedlings infected with the pathogen, 4 stages were defined for each species. The stages included untreated $V$. fordii (F0) and $V$. fordii infected with $F$. oxysporum at an early stage (F1), in the middle stage (F2) and at a late stage (F3); they also included untreated $V$. montana (M0) and V. montana infected with $F$. oxysporum at an early stage (M1), in the middle stage (M2) and at a late stage (M3). Three biological replicates were taken for each treatment. Therefore, in total, 24 RNA libraries were constructed and sequenced.

Total RNA was extracted from the plant roots and from the root-stem transition region using the EASYspin plant RNA mini kit (Aidlab, Beijing, China) and the QIAGEN RNeasy plant mini kit (QIAGEN, Valencia, CA). RNA integrity was confirmed using a Bioanalyzer 2100 and the RNA 6000 Nano Lab Chip Kit (Agilent, CA, USA). All RNA samples displayed a 260/280 ratio of greater than 2.0 and RNA integrity numbers (RIN) $\geq 7.0$. The mRNA was purified from $5 \mu \mathrm{g}$ of total RNA using oligo-dT-coupled magnetic beads (Invitrogen) and was then fragmented into small pieces using divalent cations at an elevated temperature. The cleaved RNA fragments were reversetranscribed to create the final cDNA library according to the protocol for the mRNA-seq sample preparation kit (Illumina, San Diego, USA). Finally, we performed PE sequencing on an Illumina Hiseq2000/2500 at LC-Bio Co., Ltd, Hangzhou, China following the vendor's recommended protocol.

\section{Sequence Functional Annotation and Classification}

To determine the functional annotation of the unigenes, a BLASTX search was performed against the NCBI Nr database with an $E$-value $\leq 10^{-5}$ and other databases, including SwissProt, Protein Information Resource (PIR), Protein Research Foundation (PRF) and Protein Data Bank (PDB). A BLASTN search was performed against the NCBI Nt database, GenBank, RefSeq, and PDB using a protein query. The CDS (Coding sequences) of unigenes were predicted using GENScan software. The ORFs were identified as the nucleotide sequence or as the protein translation provided by the "GetORF" program from the EMBOSS software package (Rice et al., 2000). The Blast2GO program was used to assign GO terms with an E-value $\leq$ $10^{-5}$ including molecular functions, biological processes, and cellular components (Conesa et al., 2005; Conesa and Götz, 2008). KEGG pathways were retrieved from KEGG web server (http://www.genome.jp/kegg/). The output of the KEGG analysis includes $\mathrm{KO}$ assignments and KEGG pathways that are populated with the $\mathrm{KO}$ assignments (Kanehisa et al., 2004).

\section{Identification of Orthologous Genes between $\boldsymbol{V}$. fordii and $\boldsymbol{V}$. montana}

A reciprocal-best-BLAST-hits $(\mathrm{RBH})$ method with relatively strict filters was applied to identify orthologous genes between $V$. fordii and $V$. montana based on the large-scale transcriptome sequencing. To identify and remove possible paralogous genes, all sequence pairs were first BLAST searched in GenBank by blastp, and several pairs of sequences mapped to the same protein. Next, each sequence from $V$. montana was initially searched against all sequences from $V$. fordii using Blastn, and conversely each sequence from $V$. fordii was searched against all sequences of $V$. montana. Pairs of sequences that were longer than $300 \mathrm{bp}$ and were the best hits for each other in the two species were regarded as putative orthologous genes. Finally, a total of 44,310 pairs of sequences were selected as one-to-one orthologous genes in $V$. fordii and V. montana. 


\section{Estimation of Synonymous and Non-Synonymous Substitution Rates between Orthologs}

To calculate the synonymous (Ks) and non-synonymous (Ka) substitution rate for each orthologous gene pair in $V$. fordii and $V$. montana, an e-value significance threshold of $10^{-5}$ was applied, and each member of a pair of sequences was first searched against all plant protein sequences available in GenBank using Blastx; the CDSs of each orthologous gene pair were determined based on the best alignment regions. After removing short ( $<300 \mathrm{bp}$ ) and unexpected stop codon(s)containing CDSs, the rates of synonymous and non-synonymous substitutions were estimated using the maximum likelihood method implemented in the codeml program under the F3 $\times 4$ model (Zhang et al., 2006; Langfelder and Horvath, 2008).

\section{Gene Expression Validation by Strand-Specific qPCR}

To validate the RNA-seq results in terms of gene expression, qPCR was performed using a 7500 Real-Time PCR System (Applied Biosystems, CA, USA) and a SYBR ${ }^{\circledR}$ Premix Ex Taq ${ }^{\mathrm{TM}}$ Kit (TaKaRa, Tokyo, Japan). One microgram of RNA was reverse-transcribed using SuperScript III Reverse Transcriptase (Invitrogen) for cDNA synthesis. Primers specific for Actin7a (Han et al., 2012) were used to standardize the cDNA. The genespecific primers (Supplemental Table S1) were used to amplify the corresponding genes. PCR reactions were prepared in $20 \mu \mathrm{L}$ volumes containing $2 \mu \mathrm{L}$ of 30 -fold diluted synthesized cDNA, $10 \mu \mathrm{L}$ of $2 \times \mathrm{SYBR}^{\circledR}$ Premix Ex Taq ${ }^{\mathrm{TM}}, 0.4 \mu \mathrm{L}$ of $10 \mu \mathrm{M}$ forward primer, $0.4 \mu \mathrm{L}$ of $10 \mu \mathrm{M}$ reverse primer, $0.4 \mu \mathrm{L}$ of $50 \times$ ROX reference dye and $6.8 \mu \mathrm{L}$ of sterile distilled water. The cycling conditions were set as recommended by the manufacturer $(30 \mathrm{~s}$ at $95^{\circ} \mathrm{C}$ and then 40 cycles of $95^{\circ} \mathrm{C}$ for $5 \mathrm{~s}$ and $60^{\circ} \mathrm{C}$ for $34 \mathrm{~s})$. The specificity of amplification was verified by melting curve analysis $\left(60-95^{\circ} \mathrm{C}\right)$ after 40 PCR cycles. The experiments included three biological replicates for each treatment and four technical replicates for each biological replicate and the final threshold cycle $(\mathrm{Ct})$ values were set as the mean values according to Pfaffl (2001).

\section{Data Mining}

To identify gene interactions, we applied a systems biology approach using an $\mathrm{R}$ package for weighted gene co-expression network analysis (WGCNA), which converts co-expression measures into connection weights. We selected the genes in two steps: first, we selected genes that showed significant correlation with a pathogen infection stage (false discovery rate, FDR $<0.01$, the $p$-value of Pearson's correlation efficiency was corrected by the $\mathrm{BH}$ method); from the remaining genes, we then selected the top 2000 most variable genes based on their coefficients of variance. We input the expression profiles of these selected genes to construct weighted gene co-expression modules using the WGCNA $\mathrm{R}$ package (Langfelder and Horvath, 2008). For the gene expression correlations in all of the samples of $V$. fordii and $V$. montana, the $\log _{2}$ FPKM (the expected number of fragments per kilobase of transcript sequence per millions base pairs sequenced) values for pairs of orthologous genes were calculated.

\section{RESULTS}

\section{Sister Vernicia Species Respond Differently to $F$. oxysporum}

Vernicia fordii and Vernicia montana responded differently to the pathogen $F$. oxysporum Schlecht. To characterize differences in the pathogen infection processes between $V$. fordii and $V$. montana, 4-week-old seedlings of resistant $V$. montana (M) and susceptible $V$. fordii (F) were each treated with F. oxysporum. According to the symptoms of the pathogen-infected seedlings, four stages were defined for each species. The stages included untreated $V$. montana (M0) and $V$. montana infected with F. oxysporum in the early stage (M1), in the middle stage (M2) and in the late stage (M3); they also included untreated susceptible $V$. fordii (F0) and $V$. fordii infected with $F$. oxysporum in the early stage (F1), in the middle stage (F2) and in the late stage (F3). An overview of seedlings with different treatments is shown as Figure 1.

\section{Output Statistics of the Transcriptomes of $V$. fordii and $V$. montana Inoculated with F. oxysporum}

Using Illumina $\mathrm{HiSeq}^{\mathrm{TM}} 2000$ sequencing, approximately $516,422,088$ and 559,181,176 clean paired-end (PE) reads were generated from 24 libraries of $V$. fordii (F0, F1, F2, and F3) and $V$. montana (M0, M1, M2, and M3), respectively. The number of clean reads ranged from 29.2 to 66.5 million across libraries F0, F1, F2, and F3, which were assembled together for $V$. fordii, and the read number ranged from 30.6 to 66.9 million across libraries M0, M1, M2, and M3, which were assembled for V. montana (Table 1). The high-quality reads are available in the NCBI Gene Expression Ominibus (GEO) with accession number GSE80228. De novo assembly of $V$. fordii yielded a total of 258,435 transcripts with a mean length of $1010 \mathrm{bp}$, which integrated into 133,304 unigenes with an average length of $648 \mathrm{bp}$. For $V$. montana, de novo assembly yielded a total of 245,240 transcripts with a mean length of $1376 \mathrm{bp}$, which integrated into 111,278 unigenes with an average length of $726 \mathrm{bp}$. The N50 sizes of the unigenes were 1033 and 1289 in $V$. fordii and $V$. montana, respectively (Table 2). The overall GC content of $V$. fordii in coding regions was $40.60 \%$, consistent with that of $V$. montana $(39.60 \%)$. The output statistics for sequencing and the mapping statistics for each sample stage of $V$. fordii and $V$. montana infected with F. oxysporum are shown in Supplemental Table S2.

The BLAST top-hit species distribution showed that the assembled transcript annotation in $V$. fordii had the highest homology to J. curcas (34.1\%), R. communis (14.6\%), Populus trichocarpa (5.9\%), Theobroma cacao (2.6\%), Vitis vinifera (2.5\%), Citrus sinensis (1.7\%) and others (38.6\%) (Supplemental Figure S1A). The assembled transcript annotation in $V$. montana displayed the highest homology to $R$. communis (36.2\%), Vitis vinifera (8.1\%), P. trichocarpa 


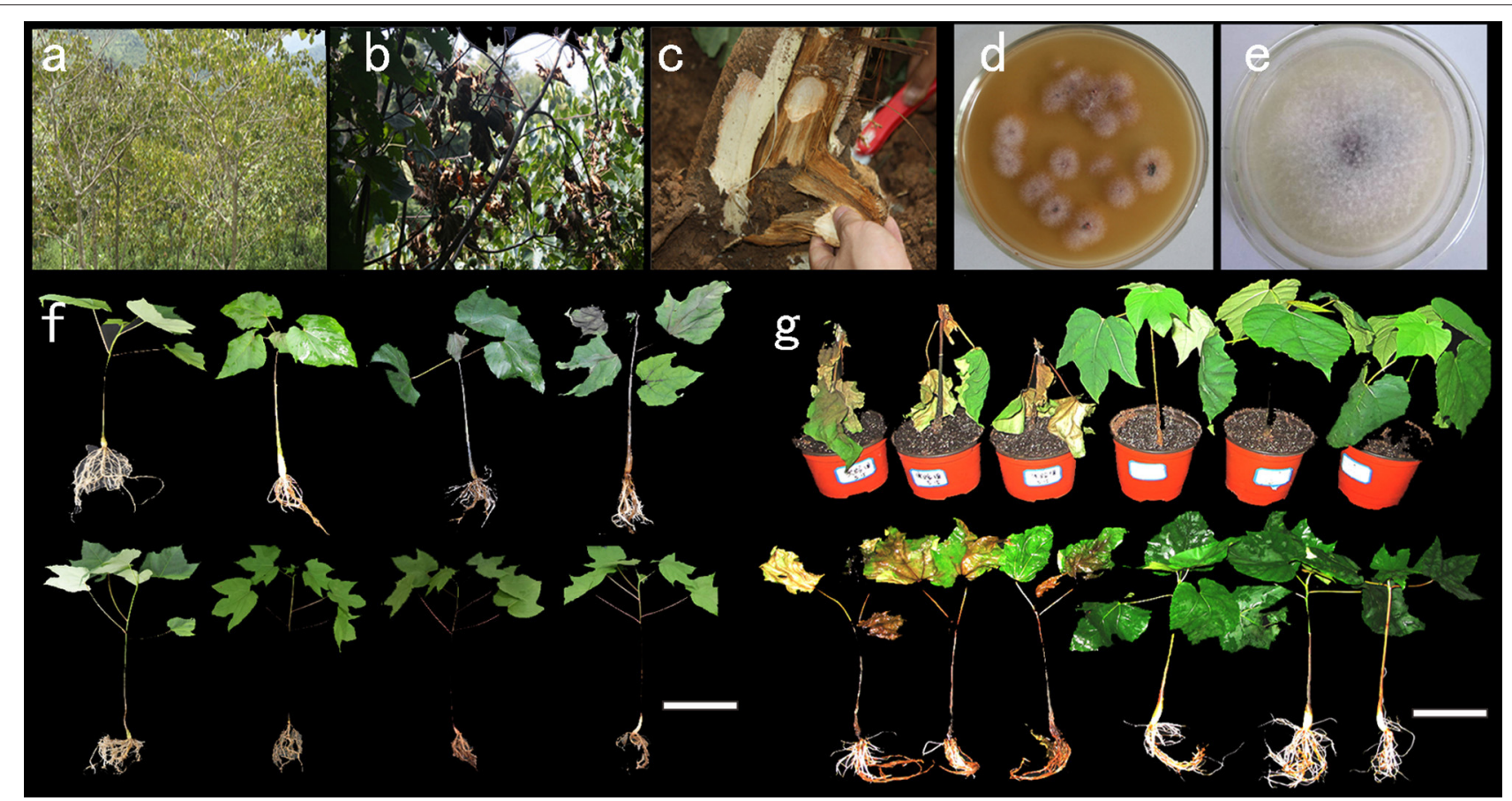

FIGURE 1 | Tung wilt pathogen isolation and pathogenicity testing in $\boldsymbol{V}$. fordii and $\boldsymbol{V}$. montana. (A) A tung forest attacked by wilt disease; (B) a $V$. fordii tree with Fusarium disease; (C) a longitudinal section of a $V$. fordii tree with wilt disease; (D) isolation of pathogens from the wilt-diseased tung tree; (E) a further pure culture of the potential fungal pathogen; (F) seedlings of $V$. fordii and $V$. montana infected with the tung wilt pathogen $F$. oxysporum at different stages: the upper row shows $V$. fordii seedlings, and the lower row shows $V$. montana seedlings; (G) seedlings of $V$. fordii and $V$. montana severely infected with $F$. oxysporum. Scale bar $=10 \mathrm{~cm}$.

TABLE 1 | Output statistics of sequencing and mapping stats for each stage samples in $V$. fordii and $V$. montana infencted with $F$. oxysporum.

\begin{tabular}{lccccccccc}
\hline & Valid data reads & Q20\% & Q30\% & GC\% & & Valid data reads & Q20\% & Q30\% & GC\% \\
\hline F0 & $47,271,474$ & 98.59 & 95.80 & 91.94 & M0 & $42,329,318$ & 98.65 & 95.63 & 91.48 \\
F0 & $66,515,356$ & 99.25 & 100 & 100 & M0 & $66,859,072$ & 99.25 & 100.00 & 100.00 \\
F0 & $29,272,386$ & 98.49 & 94.95 & 90.39 & M0 & $41,093,632$ & 98.65 & 95.08 & 90.63 \\
F1 & $31,347,672$ & 98.87 & 95.48 & 91.22 & M1 & $39,967,224$ & 97.43 & 96.37 & 93.10 \\
F1 & $63,432,696$ & 99.20 & 100 & 100 & M1 & $65,782,492$ & 99.28 & 100.00 & 100.00 \\
F1 & $29,620,658$ & 98.82 & 95.39 & 91.10 & M1 & $39,077,922$ & 98.91 & 95.35 & 91.08 \\
F2 & $29,482,698$ & 97.66 & 95.88 & 92.04 & M2 & $30,558,196$ & 99.00 & 94.76 \\
F2 & $64,285,262$ & 99.21 & 100 & 100 & M2 & $66,242,736$ & 99.33 & 100.00 & 100.00 \\
F2 & $28,992,470$ & 98.57 & 94.79 & 90.03 & M2 & $32,451,888$ & 98.55 & 95.97 & 92.26 \\
F3 & $29,419,992$ & 99.26 & 95.06 & 90.48 & M3 & $36,548,912$ & 97.97 & 95.96 & 92.27 \\
F3 & $64,236,976$ & 99.43 & 100 & 100 & M3 & $64,240,712$ & 99.28 & 100.00 & 100.00 \\
\hline
\end{tabular}

Q20: percentage is the proportion of nucleotides with a quality value $>20$ in reads.

TABLE 2 | Assembly statistics (all the clean reads from the 4 cDNA libraries of each species were together assembled).

\begin{tabular}{|c|c|c|c|c|c|c|c|c|c|c|}
\hline & & All & Median GC\% & Mean GC\% & Min length & Median length & Mean length & Max length & Total assembled bases & N50 \\
\hline \multirow[t]{2}{*}{$F$} & Unigene & 133,304 & 40.60 & 41.62 & 201 & 351 & 648 & 23,838 & $86,436,155$ & 1033 \\
\hline & Transcript & 258,435 & 40.60 & 41.62 & 201 & 558 & 1010 & 23,838 & $261,063,231$ & 1776 \\
\hline \multirow[t]{2}{*}{ M } & Unigene & 111,278 & 39.60 & 39.85 & 201 & 368 & 726 & 23,849 & $80,872,199$ & 1289 \\
\hline & Transcript & 245,240 & 39.60 & 39.85 & 201 & 801 & 1376 & 23,849 & $337,505,963$ & 2452 \\
\hline
\end{tabular}

N50: unigene length-weighted median. 
(7.9\%), T. cacao (3.8\%), C. sinensis (1.8\%) and others $(42.2 \%)$ (Supplemental Figure S1B).

For the purpose of functional annotation and classification, the coding sequences (CDS) of 67,961 (50.98\%) and 51,924 (46.66\%) unigenes were successfully predicted in the $V$. fordii and $V$. montana transcriptomes, respectively. A total of 133,304 and 111,278 unigenes in $V$. fordii and $V$. montana, respectively, were annotated from different databases. By gene ontology (GO) analysis, 46,210 (34.67\%) unigenes were assigned into 17 classes in $V$. fordii, while 36,507 (32.81\%) unigenes were classified into 19 classes in V. montana. By searching against the Kyoto Encyclopedia of Genes and Genomes Pathway database (KEGG), the unigenes were assigned to 279 KEGG pathways in both $V$. fordii and V. montana (Table 3).

\section{Vernicia Transcriptomes Display High Quality and Are Comparable between V. fordii and V. montana}

To evaluate the consistency among biological replicates, the expected number of fragments per kilobase of transcript sequence per millions base pairs sequenced (FPKM) expression data were tested by correlation analysis, and all of the correlation coefficients between biological replicates were calculated. There was a strong correlation for triplicate samples, which reached average Pearson correlations $R^{2}$ of 0.835 and 0.870 in $V$. fordii and $V$. montana, respectively (Supplemental Figure S2). Biological replicates at each sample stage clustered closely and were highly correlated.

To evaluate the conservation of gene expression patterns between $V$. fordii and $V$. montana, a reciprocal-best-BLAST-hits (RBH) method (Ward and Moreno-Hagelsieb, 2014) was applied, and the similarity threshold was set to $90 \%$. Using this method, we defined 44,310 pairs of one-to-one orthologous unigenes between the $V$. fordii $(57.60 \%, 44,310 / 76,924)$ and $V$. montana $(72.83 \%, 44,310 / 60,842)$ transcriptomes.

\section{Global Changes in Gene Expression in Resistant $V$. montana and Susceptible V. fordii}

To identify the unigenes with differential gene expression (DGE) during stages F0-F3 and M0-M3, a significance threshold of 0.05 was applied using Edge R package (Robinson et al., 2010). The differential expression modes between the two species were thus revealed (Figure 2). For V. fordii, 3002 and 6548 unigenes were upregulated and repressed, respectively, at the early stage (F1 vs. F0) after infection. At the next stage, 557 and 456 unigenes were significantly induced and repressed, respectively (F2 vs. F1). However, for V. montana, 1640 and 1786 unigenes were significantly induced and repressed, respectively, in the early stage (M1 vs. M0), while 3218 and 813 unigenes were significantly induced and repressed, respectively, at the subsequent stage (M2 vs. M1) (Figure 2, Supplemental Figure S3). It seemed that many genes responded in the early stage F1 in $V$. fordii, while a large proportion of genes was induced in the later M2 stage in V. montana.

The one-to-one orthologous gene analysis facilitated a comparison of gene expression in the two species. To investigate global changes in expression patterns, we performed $k$-means clustering of the gene expression profiles for orthologous genes in susceptible $V$. fordii and resistant $V$. montana during pathogen infection. We classified the different expression modes into 20 clusters that showed distinctive expression patterns in $V$. montana and included the corresponding orthologous genes in $V$. fordii (Figure 3, Supplemental Figure S4, and Supplemental Table S3). Our comparative transcriptome analysis revealed that a high proportion of orthologous genes exhibited similar expression patterns (Supplemental Figure S4). Only a fraction of orthologous genes, including clusters 4, 12, and 16, showed divergent expression patterns. Notably, in cluster 4 and 16, the genes showed a sharp increase in $V$. montana while exhibiting a clear decrease in $V$. fordii, thereby indicating different roles for these genes in the two species when responding to the pathogen. In cluster 1 and 9, genes exhibited dramatically increased $\left(\log _{2}\right.$ FPKM $\left.\geq 5\right)$ in $V$. montana, while expressed in an invariant way in $V$. fordii (Figure 3).

To validate the gene expression profiles, quantitative RTPCR analyses were conducted for 30 candidate genes that were chosen from different $k$-means clusters in $V$. fordii and $V$. montana (Figure 4, Supplemental Figure S5). These unigenes encoded proteins such as PR-4-like protein, chitinase, EREBP-like transcription factor, the LRR-RLK CLAVATA3, salicylic acid-binding protein 2-like, receptorlike serine/threonine kinases, cytochrome P450, alcohol dehydrogenase and osmotin, among others. The results of the qPCR analyses were generally in accordance with the gene expression profile shown in the transcriptome (Supplemental Figure S5).

The GO analysis revealed conservation across most transcripts but significant divergence between the species for a subset of RNAs. In $V$. fordii, nucleoid and protein-binding transcription factor gene expression significantly increased in the early

TABLE 3 | Transcripts and unigenes annotation statistics for the transcriptom of $V$. fordii (F) and $V$. montana (M).

\begin{tabular}{|c|c|c|c|c|c|c|c|c|}
\hline & & Total & Swiss-prot & $\mathrm{Nr}$ & Pfam & KEGG & KOG & GO \\
\hline \multirow[t]{2}{*}{$\mathrm{F}$} & Transcripts & 174,545 (67.54\%) & 117,572 (45.49\%) & $163,870(63.41 \%)$ & 143,026 (55.34\%) & 87,874 (34.00\%) & 146,484 (56.68\%) & 105,083 (40.66\%) \\
\hline & Unigenes & 76,924 (57.71\%) & $50,220(37.67 \%)$ & 63,225 (47.43\%) & 60,310 (45.24\%) & 39,367 (29.53\%) & 44,074 (33.06) & $46,210(34.67)$ \\
\hline \multirow[t]{2}{*}{ M } & Transcripts & $175,571(71.59 \%)$ & $108,050(44.06 \%)$ & $172,292(70.26 \%)$ & $140,300(57.21 \%)$ & 165,367 (67.43\%) & $143,551(58.53 \%)$ & 95,803 (39.06\%) \\
\hline & Unigenes & 60,842 (54.68\%) & 40,013 (35.96\%) & 53,395 (47.98\%) & 47,593 (42.77\%) & 29,847 (26.82\%) & 34,528 (31.03\%) & 36,507 (32.81\%) \\
\hline
\end{tabular}



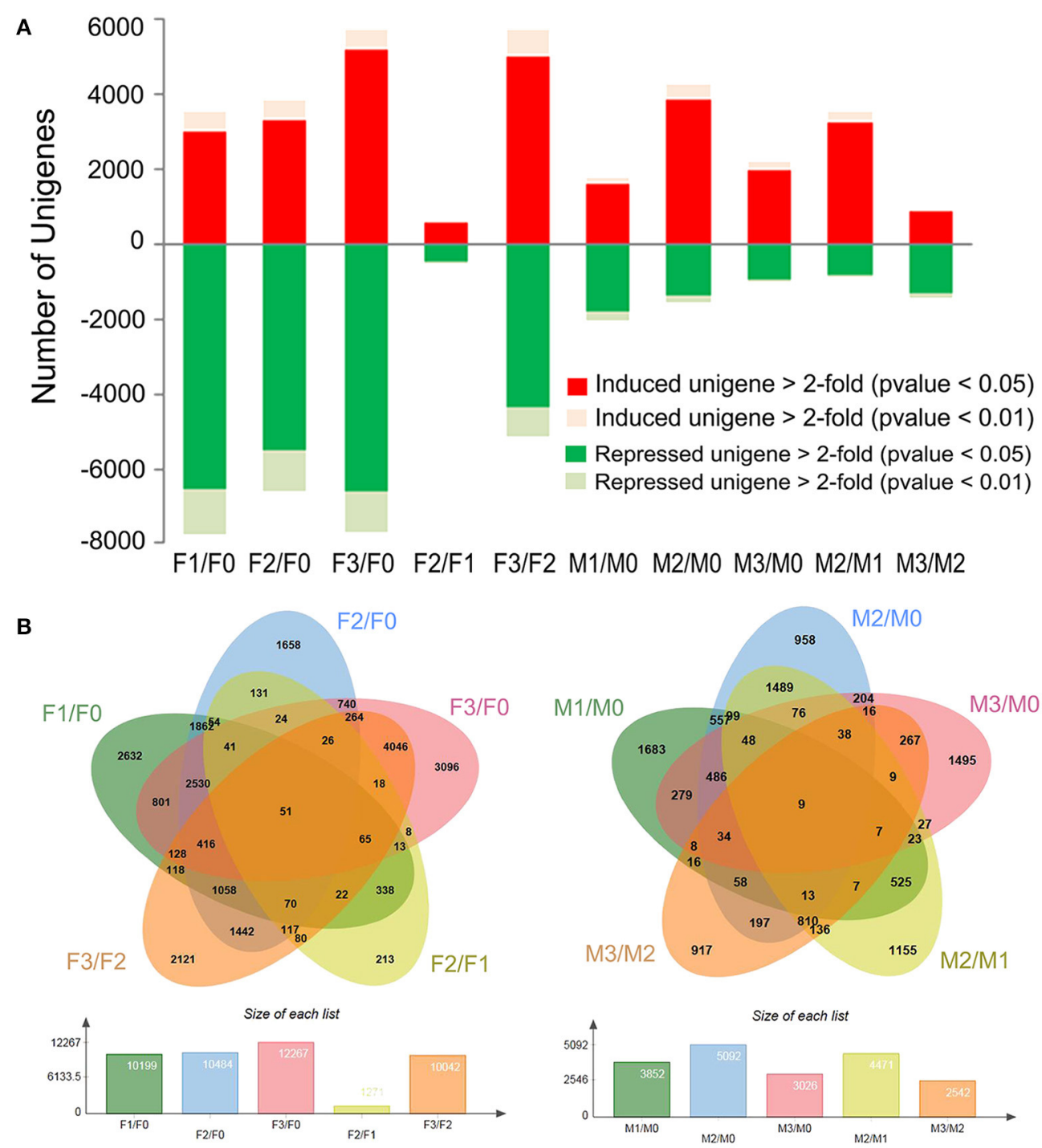

FIGURE 2 | Global changes in gene expression in resistant $\boldsymbol{V}$. montana and susceptible $\boldsymbol{V}$. fordii during $\boldsymbol{F}$. oxysporum infection. (A) The number of >2-fold induced and repressed unigenes ( $p<0.05$ and 0.01$)$ for F1 vs. F0, F2 vs. F1 and F3 vs. F2 and for M1 vs. M0, M2 vs. M1 and M3 vs. M2; (B) the numbers of commonly and specifically induced and repressed unigenes at different stages of $F$. oxysporum infection in the two species are shown in the overlapping and non-overlapping regions, respectively.
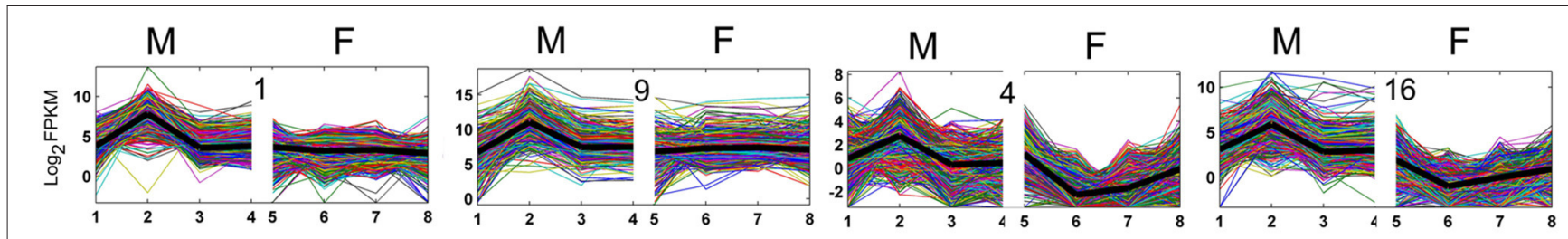

FIGURE 3 | Clustering of significantly differential expressed orthologous genesusing $\boldsymbol{k}$-means clustering method according to the gene expression profiles in $\boldsymbol{V}$. montana (M) and $\boldsymbol{V}$. fordii $(\mathbf{F})$ infected with the pathogen $\boldsymbol{F}$. oxysporum. The differential expression modes were classified into twenty clusters in V. montana, and the corresponding orthologous genes in V. fordii were analyzed for their expression modes (Supplemental Figure S4).

response stage. In V. montana, receptor activity and protein tag genes showed strong induction in the early stage. Our study generated a substantial number of transcript sequences during the infection responses of resistant and susceptible Vernicia species. The results contributed to the identification of pathways and functional genes in Vernicia species that respond to pathogen 

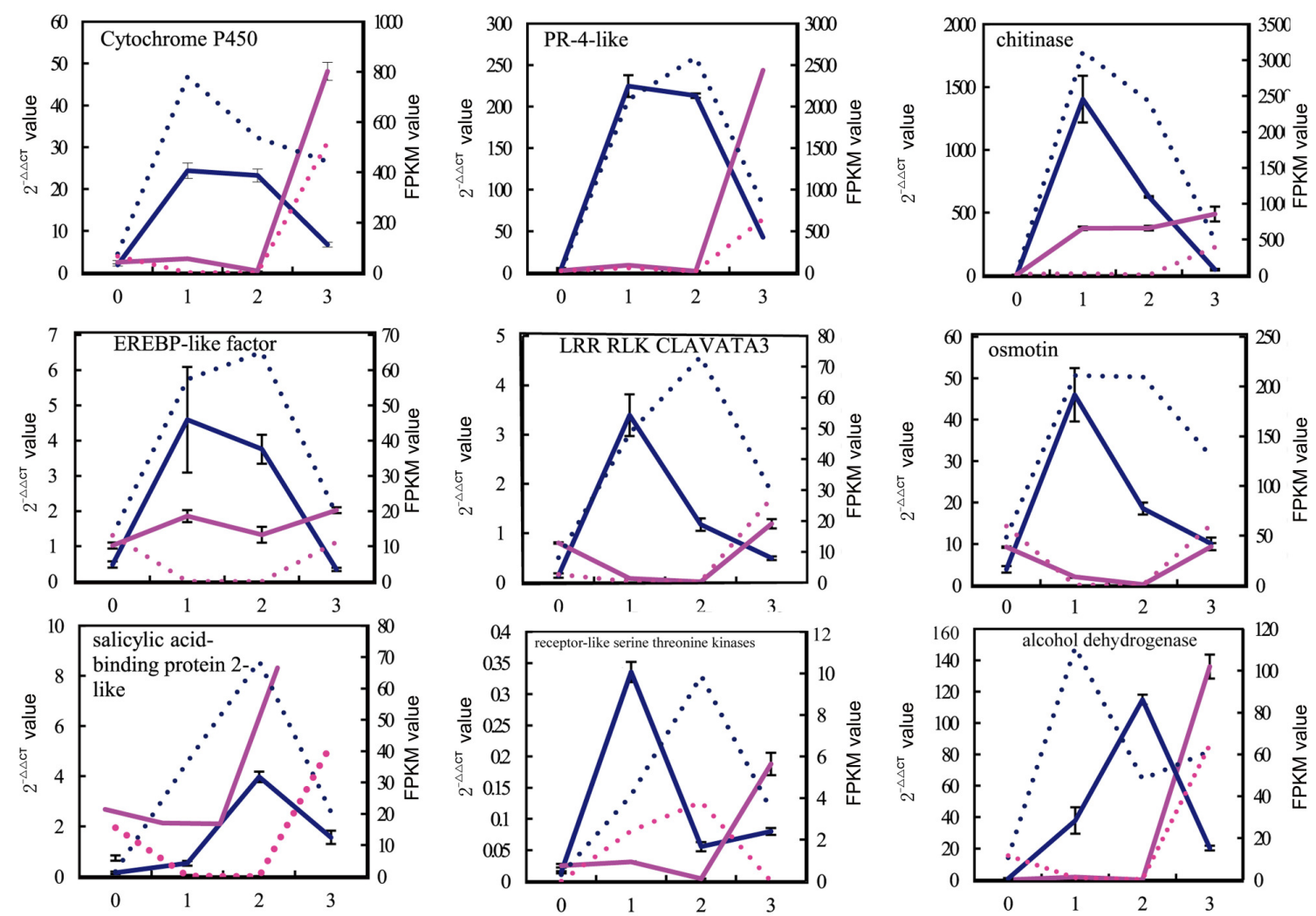

FIGURE 4 | Verification of the expression profiles of 9 pairs of unigenes by qPCR. In total, 30 candidate genes were chosen to validate using qPCR from among the different k-means clusters in V. fordii and $V$. montana (Supplemental Figure S4). The results of qPCR were generally in accordance with the gene expression profiles in the transcriptome data. $X$-axis represented the different stage of $F$. oxysporum infection. 0 , the stage before infection; 1 , the early stage of infection with F. oxysporum; 2; the middle stage of infection with F. oxysporum; 3 , the late stage of infection with F. oxysporum. The left Y-axis represented the expression level $\left(2^{-\Delta \Delta C T}\right.$ value) of genes. The right Y-axis represented the expression level of genes according to the FPKM value. The blue solid line represented the expression level $\left(2^{-\triangle \Delta C T}\right)$ of genes in $V$. montana (M) using QPCR; the blue dotted line represented the expression level of genes in $V$. montana (M) according to the FPKM value; the red real line represented the expression level $\left(2^{-\Delta \Delta C T}\right.$ ) of genes in $V$. fordii (F) using qPCR; the red dotted line represented the expression level of genes in $V$. fordii (F) according to the FPKM value.

attack, and they will aid in understanding the mechanisms with which $V$. fordii and $V$. montana respond to F. oxysporum.

\section{DISCUSSION}

\section{Conserved and Divergent Expression Patterns between $V$. fordii and $V$. montana When Responding to F. oxysporum}

To reveal genes that were highly conserved or divergent in response to $F$. oxysporum infection, we performed correlation analysis between orthologous genes. In total, we identified 450 unigenes that were significantly connected (either conserved or divergent, with correlation coefficient cutoffs of $r>0.65$ or $r<-0.65, p<0.05)$ between the two species; they are listed in Supplemental Table S4. Among them, 157 unigenes were significantly negatively associated $(r<-0.65, p<0.05)$; they included two Myb transcription factors, mitogen-activated protein kinase 3 (MAPK3), a homeobox-leucine zipper protein, a G-box-binding factor, RIO kinase 2, phytochrome-interacting factor 3 (PIF3) and others. Several Mybs reportedly participate in defending against pathogen attack, including AtMyb30, AtBOS1 (AtMyb108), and TaPIMP1 (Vailleau et al., 2002; Zhang et al., 2012; Corso et al., 2015), yet the associated regulatory mechanisms and signaling processes remain largely unknown. The expression of MAPK3 and a homeobox-leucine zipper protein increased in the later stage of the response to cadmium stress (Yang et al., 2015). A homeobox-leucine zipper gene from sunflower and Arabidopsis conferred drought and salt tolerance (Shin et al., 2004; Dezar et al., 2005). The G-box is the cognate cis-element for the bZIP, bHLH, and NAC proteins. bHLH is reportedly a critical protein in plant development, senescence, iron metabolism and regulation of pathogen defense responses, and it leads to the activation of the salicylic acid signaling pathway (Zhang et al., 2013; Aparicio and Pallás, 2016). PIF3 activates light-responsive transcriptional network genes in coordination with the circadian clock and plant hormones to modulate plant growth and development. RIF3 is also a candidate target for manipulating the weed stress response in soybeans, and it regulates the plant response to drought and salt stress in maize (Gao et al., 2015; Horvath et al., 2015). 
We found that 293 unigenes showed a significant positive association in their expression in the two species using a two-sample $t$-test (with correlation coefficients of $r>0.65$, $p<0.05$; Supplemental Table S4); they included EREBP transcription factors, serine/threonine protein kinases, a receptor-like serine/threonine protein kinase, MAPKKKs, a proline-rich receptor-like protein kinase, a cytochrome P450 and others. EREBP was reported to confer biotic and abiotic stress tolerance in rice (Jisha et al., 2015). Serine/threonine protein kinases and receptor-like serine/threonine protein kinases have been associated with the early stage of plant defense.

To functionally classify the 450 significantly correlated genes, we applied REVIGO and ggplot2 and observed significant enrichment of these genes in multiple GO categories (Supek et al., 2011). The GO enrichment analysis of the 450 pairs of unigenes with significant positive or negative correlations is shown in Figure 5. The significantly positively correlated GO terms among biological processes included fungal-type cell wall beta-glucan metabolic process, defense response and small GTPase mediated signal transduction, among others (Figure 5A). The significantly positively correlated GO terms among molecular functions included alcohol dehydrogenase (NADP+) activity, (-)-menthol dehydrogenase activity, binding, protein binding, ATP binding and structural constituents of the ribosome (Figure 5B). The significantly negatively correlated GO terms among biological processes included translation, isocitrate metabolic process, regulation of chromatin silencing at telomere and small GTPase mediated signal transduction, among others (Figure 5C). The significantly negatively correlated GO terms for molecular function included methyl jasmonate esterase activity, methyl salicylate esterase activity and isocitrate dehydrogenase (NADP+) activity (Figure 5D).
A

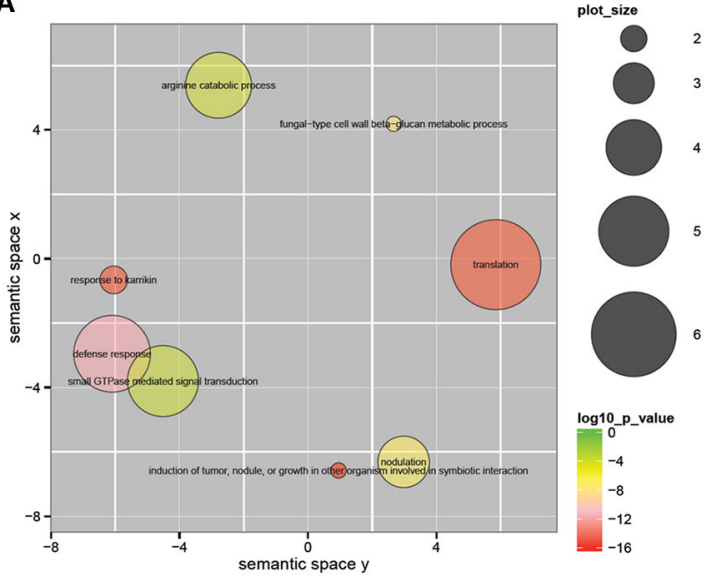

C
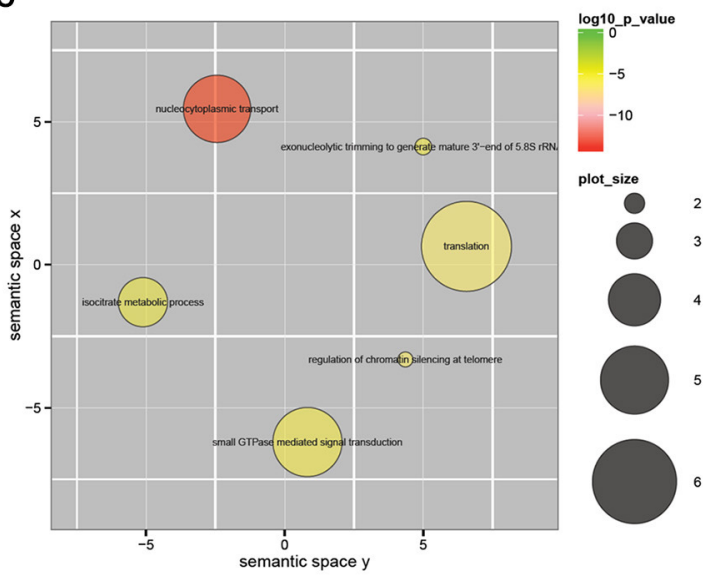

B

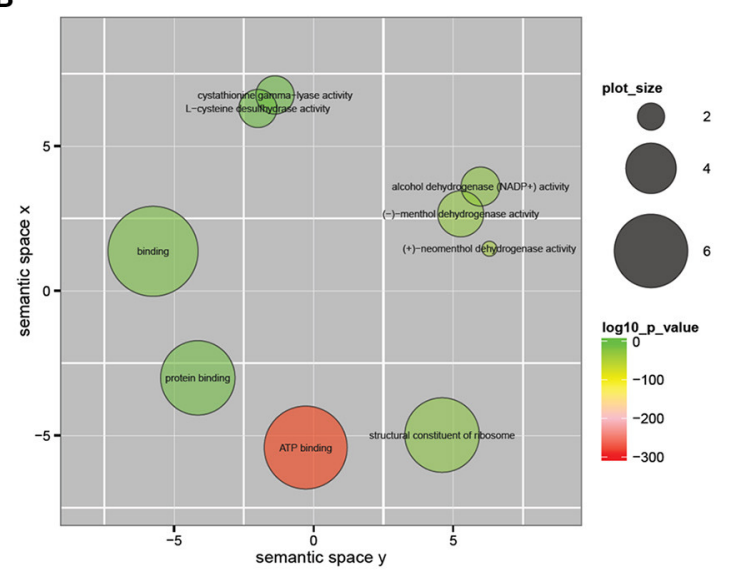

D

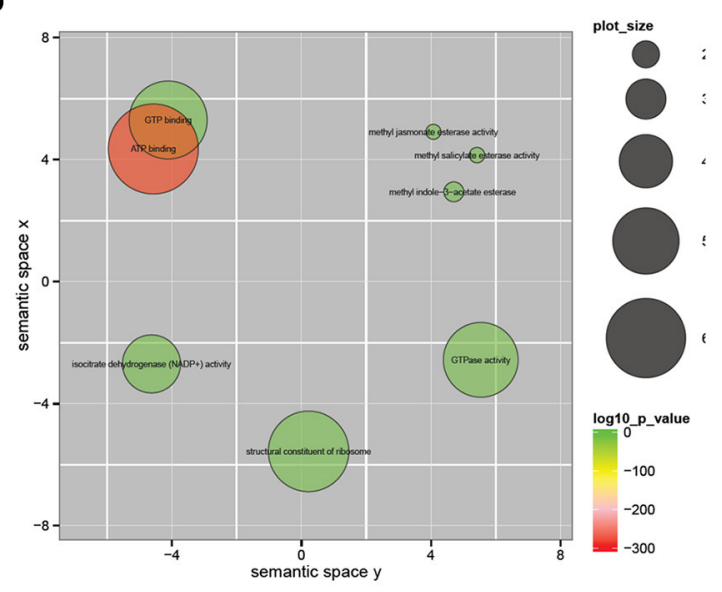

FIGURE 5 | Gene ontology enrichment analysis of $\mathbf{4 5 0}$ pairs of unigenes that showed significant positive or negative correlation (correlation coefficients $\boldsymbol{r}>\mathbf{0 . 6 5}$ or $\boldsymbol{r}<-\mathbf{0 . 6 5}, \boldsymbol{p}<\mathbf{0 . 0 5}$ ) between the gene expression modes in $\boldsymbol{V}$. fordii and $\boldsymbol{V}$. montana. (A) Significantly positively correlated GO term in biological process; (B) significantly positively correlated GO term in molecular function; (C) significantly negatively correlated GO term in biological process; (D) significantly negatively correlated GO term in molecular function. The scatterplot shows the cluster representatives in a two dimensional space derived by applying multidimensional scaling to a matrix of the GO terms' semantic similarities. Bubble color indicates the log 10 p-value (legend in upper right-hand corner); plot size indicates the frequency of the GO term in the underlying GOA database (bubbles of more general terms are larger). 


\section{Gene Co-expression Networks and Pathways in Resistant V. montana}

To give a higher-order interpretation of gene expression relationships and to identify modules of co-expressed genes that were functionally related, we implemented a systems biology approach and analyzed the gene expression profiles of 24 susceptible and resistant transcriptomes. The centralized genes, called hub genes, were highly connected with peripheral genes. We raised the weighted cutoff value to $>0.50$ to identify hub genes with the strongest connections to other genes. Under this criterion, we visualized the 50 most highly connected genes as hub genes. Among them, 26 hub genes were functionally annotated as hypothetical proteins, and 17 unigenes playing essential roles in plant pathogen resistance (Supplemental Table S5). We further used the software Cytoscape 3.2.1 to build the co-expressed gene network (Figure 6A). As the Figure 6A showed, among the top 50 hub genes, the serine/threonine protein kinase
D6PK and the LRR-RLK CLAVATA2 were strongest, with 2611 and 1752 connections, respectively (Figure 6A). LRRRLKs are reportedly involved in plant defense responses against various classes of pathogens and may initiate the activation of the MAPK pathway, WRKY transcription factors, ion channels and the NADPH oxidase complex (Yang et al., 2012). The LRR-RLK2 CLAVATA has been reported to confer enhanced disease resistance to bacterial wilt (Hanemian et al., 2016). Diacylglycerol kinase (DGK), which confers resistance to Magnaporthe grisea (Zhang et al., 2008) was also identified as a hub gene with 1253 connections. Furthermore, glycosyltransferase (Gts), which is reportedly involved in resistance to F. oxysporum (LorencKukuła et al., 2009), was also identified as a hub gene with 185 connections. The homeobox-leucine zipper protein GLABRA2 was recognized as a hub gene with 848 connections, and the EREBP transcription factor was defined as a hub gene with 666 connections.
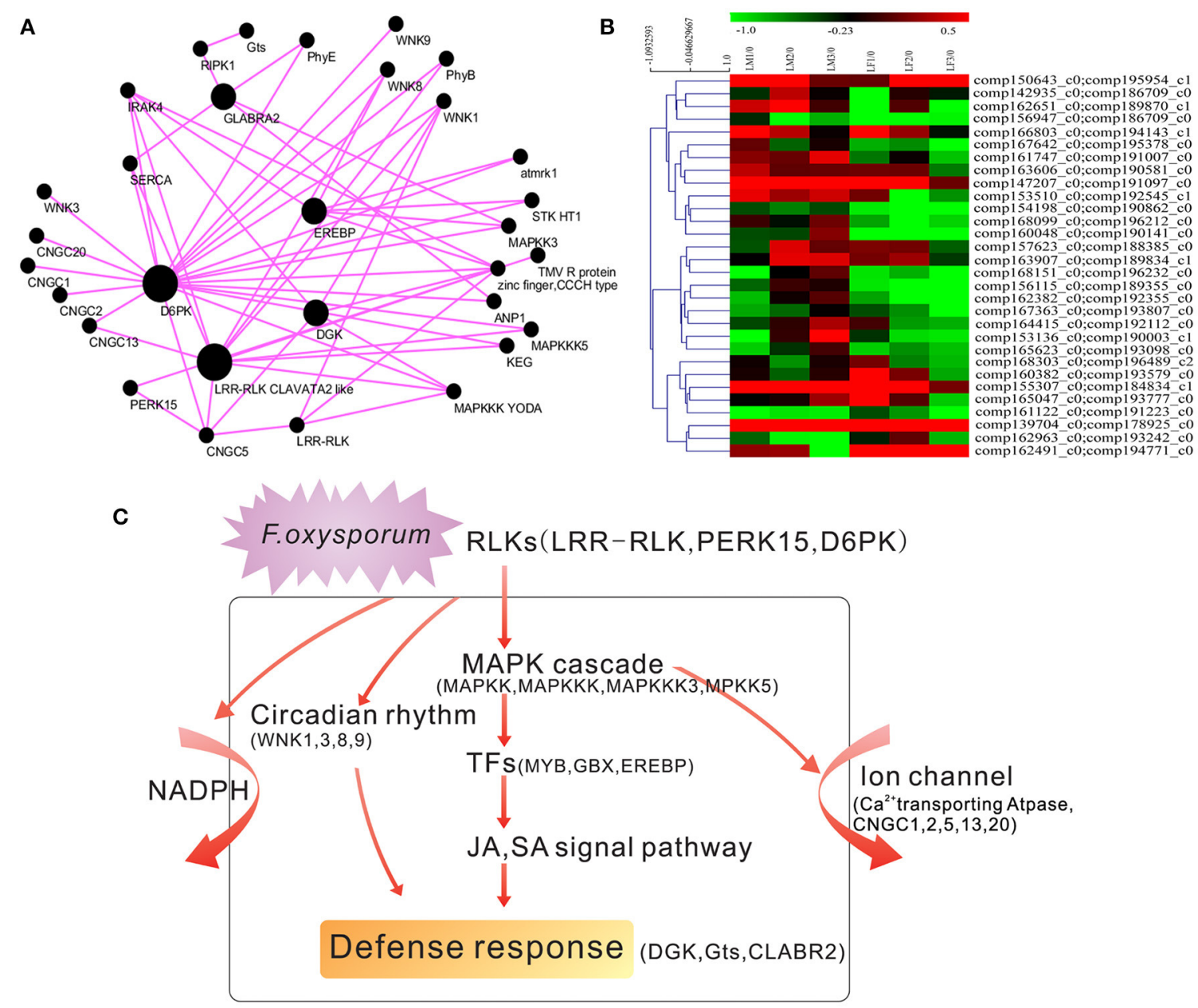

FIGURE 6 | Hub gene identification and model for the defense response in $\mathbf{V}$. montana. (A) ldentification of resistance-related hub genes in $V$. montana responding to F. oxysporum. The connections were drawn using Cytoscape. The genes with a weighted cutoff value $>0.50$ are shown. Each node represents a gene. Black nodes indicate hub genes. Bigger nodes indicate more connections. (B) Expression patterns of pairs of hub genes in the two species during infection with F. oxysporum. (C) A model to describe the major molecular reactions underlying the defense responses to $F$. oxysporum in resistant $V$. montana. 
Among the top 50 hub genes, the expression modes of Gts, the proline-rich receptor-like protein kinase PERK15 and an EREBP-like factor in $V$. fordii and $V$. montana were significantly and positively correlated during the infection (correlation coefficient $r>0.65, p<0.05$ ). However, the expression of the homeobox-leucine zipper protein GLABRA2 was significantly negatively correlated between the two species (correlation coefficient $r<-0.65, p<0.05)$. Genes with higher connectivity tended to have a stronger correlation with resistance to $F$. oxysporum, suggesting a potentially important role of highly connected genes (hub genes) in the resistance of $V$. montana to F. oxysporum.

The expression patterns of the hub genes were analyzed in the two species during infection with $F$. oxysporum according to their FPKM values. The expression of 30 of hub genes showed induction in $V$. montana but repression in $V$. fordii during infection with the pathogen F. oxysporum (Figure 6B).

To functionally classify these significant genes, we observed the enrichment of these genes in several KEGG pathways. Significant pathways included the MAPK signaling pathway, plant-pathogen interaction, circadian rhythm, the calcium signaling pathway and apoptosis. Hierarchical clustering revealed major modules that exhibited totally different expression patterns during the infection process. Enrichment for these categories indicated that the KEGG pathways described above play a major role in driving transcriptional variation among Vernicia species infected with F. oxysporum.

\section{Evolution Analysis Reveal Conservation and Diversification between Vernicia Orthologs}

To define the molecular evolution of orthologous genes shared by $V$. fordii and $V$. montana, particularly genes undergoing purifying or positive selection, we calculated the ratio of synonymous (Ks) and non-synonymous (Ka) substitution rates $(\mathrm{Ka} / \mathrm{Ks})$ for each pair of orthologous genes (Yang and Bielawski, 2000). $\mathrm{Ka} / \mathrm{Ks}=1$ signifies neutral evolution, $\mathrm{Ka} / \mathrm{Ks}>1$ is a sign of positive (adaptive) selection, and $\mathrm{Ka} / \mathrm{Ks}<1$ is a sign of negative (purifying) selection. Our results showed that the mean value of $\mathrm{Ka} / \mathrm{Ks}$ for orthologous gene pairs was 3.32. The majority of sequences $(90.17 \%, 6020 / 6676)$ had a $\mathrm{Ka} / \mathrm{Ks}$ ratio of $<1$, while a small portion of genes $(9.83 \%, 656 / 6676)$ had a $\mathrm{Ka} / \mathrm{Ks}$ ratio of $>1$, suggesting that most of the orthologous genes are undergoing purifying selection. We also identified 208 sequences under accelerated evolution, with ratios of $\mathrm{Ka} / \mathrm{Ks}$ $>1$ and $p$-value $<0.05$, among which 167 sequences showed ratios of $\mathrm{Ka} / \mathrm{Ks}>2$ and $p$-value $<0.05$ (Supplemental Table S6). These fast-evolving transcripts may be useful for identifying genes that perhaps strongly underwent positive selection during the evolutionary process and that may be responsible for speciation in the Camellia lineage. Notably, the proline-rich receptor-like protein kinase PERK15 was not only significantly positively correlated between $V$. fordii and $V$. montana during $F$. oxysporum infection, but it also had a $\mathrm{Ka} / \mathrm{Ks}$ ratio of $>1$.

\section{Hypothesized Molecular Reactions in Resistant V. montana Responding to F. oxysporum}

F. oxysporum, a hemi-biotrophic root pathogen, infects a number of plants, including cotton, tomato and banana. Some Arabidopsis mutants with impaired jasmonic acid (JA) signaling are more susceptible to other pathogens but show strong resistance to $F$. oxysporum (Anderson et al., 2004; Kidd et al., 2009). This result suggests that $F$. oxysporum disguises itself as a beneficial microbe, activating the JA pathway to gain entry into the cell as a hemi-biotrophic pathogen (Chen et al., 2014a).

The LRR-RLK2 CLAVATA was defined as a main hub gene in our study. Thus, hierarchical clustering of the expression modes of all pairs of LRR-RLKs in $V$. fordii and V. montana was conducted (Supplemental Figure S6). At the bottom of the hierarchical clustering, the expression of a small set of LRR-RLKs was induced in $V$. montana but repressed in $V$. fordii. LRR-RLKs have been reported to have key roles in pathogen recognition, and they may lead to the activation of ion channels, the NADPH oxidase complex and MAPK cascades after trans-phosphorylation and phosphorylation events (Yang et al., 2012). Similarly, we conducted gene enrichment analysis on 450 unigenes and also clearly identified the above pathways (Figure 5). Moreover, enrichment analysis of the top genes indicated roles for the MAPK signaling pathway, plantpathogen interaction, circadian rhythm, the calcium signaling pathway and apoptosis during $F$. oxysporum infection of $V$. montana. Based on our results, we propose a model to describe the major molecular reactions that underlie the defense responses of resistant $V$. montana to $F$. oxysporum (Figure 6C). Upon attack by $F$. oxysporum, protein kinases, such as LRR-RLK CLAVATA, PERK15 and D6PK, act as a first line of defense against the pathogen. A series of signal transduction pathways, including the MAPK, JA, and SA signaling pathways, are then initiated. Meanwhile, circadian rhythms, NADPH and ion channels are activated by receptor like kinases (RLKs) in the plasma membrane. Furthermore, transcription factors, such as Myb, GBS, and EREBP, are activated, thereby finally inducing the expression of a series of defense-related genes, including DGX, Gts, CLABR2, and others. There appears to be some crosstalk in the signaling pathways.

\section{ACCESSION NUMBERS}

The datasets supporting the conclusions of this article are available in the NCBI GEO repository under accession number GSE80228. http://www.ncbi.nlm.nih.gov/gds/

\section{AUTHOR CONTRIBUTIONS}

YC conceived the original screening and research plans; YW supervised the experiments; YC performed most of the experiments; MG, HZ, and QZ provided technical assistance to YC; YC, HY designed the experiments and analyzed the data; YC conceived the project and wrote the article with contributions 
of all the authors; HY, YW supervised and complemented the writing.

\section{ACKNOWLEDGMENTS}

The work was financially supported by the National Natural Science Foundation of China (31200485) and the Special Fund for Forestry Science Research in the Public Interest (201304102).

\section{SUPPLEMENTARY MATERIAL}

The Supplementary Material for this article can be found online at: http://journal.frontiersin.org/article/10.3389/fpls.2016. 01974/full\#supplementary-material

Supplemental Figure S1 | The top BLAST species hits with homology to

V. fordii (A) and V. morntana (B) according to the transcript annotation.

Supplemental Figure S2 | Pearson's correlation heat map of the unigenes (FPKM) of all the samples $(\mathrm{n}=3)$ in V. fordii and V. montana. F, V. fordii; M, V. Montana.

Supplemental Figure S3 | A volcano plot using a fold change difference for differential expressed genes in $V$. fordii and $V$. montana.

Supplemental Figure S4 | A high proportion of orthologous genes exhibited similar expression patterns using $k$-means clustering of one-to-one orthologous unigene pairs according to the gene expression profiles in $\boldsymbol{V}$. montana (M) and $\boldsymbol{V}$. fordii $(\mathrm{F})$ infected with the pathogen F. oxysporum. The differential expression modes were classified into 20 clustersin V. montana, and the corresponding orthologous genes in $V$. fordii were analyzed for their expression modes (the other four clusters expressed significantly different were shown in Figure 3).

\section{REFERENCES}

Anderson, J. P., Badruzsaufari, E., Schenk, P. M., Manners, J. M., Desmond, O. J., Ehlert, C., et al. (2004). Antagonistic interaction between abscisic acid and jasmonate-ethylene signaling pathways modulates defense gene expression and disease resistance in Arabidopsis. Plant Cell 16, 3460-3479. doi: $10.1105 /$ tpc. 104.025833

Aparicio, F., and Pallás, V. (2016). The coat protein of Alfalfa mosaic virus interacts and interferes with the transcriptional activity of the bHLH transcription factor ILR3 promoting salicylic-dependent defense signaling response. Mol. Plant Pathol. doi: 10.1111/mpp.12388. [Epub ahead of print].

Brown, K., and Keeler, W. (2005). The history of tung oil. Wildland Weeds 9, 4-6.

Catanzariti, A. M., Lim, G. T., and Jones, D. A. (2015). The tomato I-3 gene: a novel gene for resistance to Fusarium wilt disease. New Phytol. 207, 106-118. doi: $10.1111 /$ nph. 13348

Chen, Y. C., Kidd, B. N., Carvalhais, L. C., and Schenk, P. M. (2014a). Molecular defense responses in roots and the rhizosphere against Fusarium oxysporum. Plant Signal. Behav. 9:e977710. doi: 10.4161/15592324.2014.977710

Chen, Y. C., Wong, C. L., Muzzi, F., Vlaardingerbroek, I., Kidd, B. N., and Schenk, P. M. (2014b). Root defense analysis against Fusarium oxysporum reveals new regulators to confer resistance. Sci. Rep. 4:5584. doi: 10.1038/srep05584

Chen, Y. H., Chen, J. H., Chang, C. Y., and Chang, C. C. (2010). Biodiesel production from tung (Vernicia fordii) oil and its blending properties in different fatty acid compositions. Bioresource Technol. 101, 9521-9526. doi: 10.1016/j.biortech.2010.06.117

Conesa, A., and Götz, S. (2008). Blast2GO: A comprehensive suite for functional analysis in plant genomics. Int. J. Plants Genom. 2008:619832. doi: $10.1155 / 2008 / 619832$

Conesa, A., Götz, S., García-Gómez, J. M., Terol, J., Talón, M., and Robles, M. (2005). Blast2GO: a universal tool for annotation, visualization and
Supplemental Figure S5 | Verification of the expression profiles of 21 pairs of unigenes by real-time quantitative RT-PCR. $X$-axis represented the different stage of $F$. oxysporum infection. 0 , the stage before infection; 1 , the early stage of infection with F. oxysporum; 2; the middle stage of infection with F. oxysporum; 3 , the late stage of infection with F. oxysporum. The left Y-axis represented the expression level $\left(2^{-\Delta \Delta C T}\right.$ value) of genes. The right $Y$-axis represented the expression level of genes according to the FPKM value. The blue solid line represented the expression level $\left(2^{-\Delta \Delta C T}\right)$ of genes in $V$. montana $(M)$ using qPCR; the blue dotted line represented the expression level of genes in $V$. montana (M) according to the FPKM value; the red real line represented the expression level $\left(2^{-\Delta \Delta C T}\right)$ of genes in $V$. fordii (F) using qPCR; the red dotted line represented the expression level of genes in $V$. fordii $(F)$ according to the FPKM value.

Supplemental Figure S6 | Comparative expression patterns of pairs of LRR-RLK genes between susceptible $V$. fordii and resistant $V$. montana underlying the response to attack by $F$ oxysporum.

Supplemental Table S1 | Primer sequences of genes amplification and qPCR in the study.

Supplemental Table S2 | Output statistics of sequencing and mapping stats for each sample stage in $V$. fordii and $V$. montana infected with F. oxysporum.

Supplemental Table S3 | Statistical analysis on the $\mathbf{k}$ mean clusters of orthologous genes in $V$. fordii and $V$. montana.

Supplemental Table S4 | The list of genes with significantly correlated expression between $V$. fordii and $V$. montana using a two-sample $t$-test (correlation coefficient $r>0.65$ or $r<-0.65, p<0.05$ ).

Supplemental Table S5 | Identification of the top $\mathbf{5 0}$ hub genes with a raised weighted cutoff value $>0.50$.

Supplemental Table S6 | The molecular evolution of orthologous genes between $V$. fordii and $V$. montana under accelerated evolution with ratios of $\mathrm{Ka} / \mathrm{Ks}>1$ and $p<0.05$.

analysis in functional genomics research. Bioinformatics 21, 3674-3676 doi: 10.1093/bioinformatics/bti610

Corso, M., Vannozzi, A., Maza, E., Vitulo, N., Meggio, F., Pitacco, A., et al. (2015). Comprehensive transcript profiling of two grapevine rootstock genotypes contrasting in drought susceptibility links the phenylpropanoid pathway to enhanced tolerance. J. Exp. Bot. 66, 5739-5752. doi: 10.1093/jxb/e rv274

Dezar, C. A., Gago, G. M., Gonzalez, D. H., and Chan, R. L. (2005). Hahb-4, a sunflower homeobox-leucine zipper gene, is a developmental regulator and confers drought tolerance to Arabidopsis thaliana plants. Transgenic Res. 14, 429-440. doi: 10.1007/s11248-005-5076-0

Gao, Y., Jiang, W., Dai, Y., Xiao, N., Zhang, C., Li, H., et al. (2015). A maize phytochrome-interacting factor 3 improves drought and salt stress tolerance in rice. Plant Mol. Biol. 87, 413-428. doi: 10.1007/s11103-015-0288-Z

Han, X., Lu, M., Chen, Y., Zhan, Z., Cui, Q., and Wang, Y. (2012). Selection of reliable reference genes for gene expression studies using realtime PCR in tung tree during seed development. PLoS ONE 7:e43084. doi: 10.1371/journal.pone.0043084

Hanemian, M., Barlet, X., Sorin, C., Yadeta, K. A., Keller, H., Favery, B., et al. (2016). Arabidopsis CLAVATA1 and CLAVATA2 receptors contribute to Ralstonia solanacearum pathogenicity through a miR169-dependent pathway. New Phytol. 211, 502-515. doi: 10.1111/nph.13913

Horvath, D. P., Hansen, S. A., Moriles-Miller, J. P., Pierik, R., Yan, C., Clay, D. E., et al. (2015). RNAseq reveals weed-induced PIF3-like as a candidate target to manipulate weed stress response in soybean. New Phytol. 207, 196-210. doi: $10.1111 /$ nph.13351

Jisha, V., Dampanaboina, L., Vadassery, J., Mithöfer, A., Kappara, S., and Ramanan, R. (2015). Overexpression of an AP2/ERF type transcription factor OsEREBP1 confers biotic and abiotic stress tolerance in rice. PLOS ONE 10;e0127831. doi: 10.1371/journal.pone.0127831 
Kanehisa, M., Goto, S., Kawashima, S., Okuno, Y., and Hattori, M. (2004). The KEGG resource for deciphering the genome. Nucleic Acids Res. 32, D277-D280. doi: 10.1093/nar/gkh063

Kidd, B. N., Edgar, C. I., Kumar, K. K., Aitken, E. A., Schenk, P. M., Manners, J. M., et al. (2009). The mediator complex subunit PFT1 is a key regulator of jasmonate-dependent defense in Arabidopsis. Plant Cell. 21, 2237-2252. doi: $10.1105 /$ tpc. 109.066910

Langfelder, P., and Horvath, S. (2008). WGCNA: an R package for weighted correlation network analysis. BMC Bioinf. 9:e559. doi: 10.1186/1471-2105-9-559

Lorenc-Kukuła, K., Zuk, M., Kulma, A., Czemplik, M., Kostyn, K., Skala, J., et al. (2009). Engineering flax with the GT family 1 Solanum sogarandinum glycosyltransferase SsGT1 confers increased resistance to Fusarium infection. J. Agric. Food Chem. 57, 6698-6705. doi: 10.1021/jf900833k

Ma, L. J., van der Does, H. C., Borkovich, K. A., Coleman, J. J., Daboussi, M. J., Di Pietro, A., et al. (2010). Comparative genomics reveals mobile pathogenicity chromosomes in Fusarium. Nature 464, 367-373. doi: 10.1038/nature08850

Mahdavi, F., Sariah, M., and Maziah, M. (2012). Expression of rice thaumatin-like protein gene in transgenic banana plants enhances resistance to fusarium wilt. Appl. Biochem. Biotechnol. 166, 1008-1019. doi: 10.1007/s12010-011-9489-3

Michielse, C. B., and Rep, M. (2009). Pathogen profile update: Fusarium oxysporum. Mol. Plant Pathol. 10, 311-324. doi: 10.1111/j.1364-3703.2009.00538.x

Park, J. Y., Kim, D. K., Wang, Z. M., Lu, P., Park, S. C., and Lee, J. S. (2008). Production and characterization of biodiesel from tung oil. Appl. Biochem. Biotechnol. 148, 109-117. doi: 10.1007/s12010-007-8082-2

Pfaffl, M. W. (2001). A new mathematical model for relative quantification in real-time RT-PCR. Nucleic Acids Res. 29, e45. doi: 10.1093/nar/29.9.e45

Rice, P., Longden, I., and Bleasby, A. (2000). EMBOSS: the European molecular biology open software suite. Trends Genet 16, 276-277. doi: 10.1016/S0168-9525(00)02024-2

Robinson, M. D., McCarthy,. D. J., and Smyth, G. K. (2010). edgeR: bioconductor package for differential expression analysis of digital gene expression data. Bioinformatics 26, 139-140. doi: 10.1093/bioinformatics/btp616

Shang, Q., Jiang, W., Lu, H., and Liang, B. (2010). Properties of Tung oil biodiesel and its blends with 0\# diesel. Bioresource Technol. 101, 826-828. doi: 10.1016/j.biortech.2009.08.047

Shin, D., Koo, Y. D., Lee, J., Lee, H. J., Baek, D., Lee, S., et al. (2004). Athb-12, a homeobox-leucine zipper domain protein from Arabidopsis thaliana, increases salt tolerance in yeast by regulating sodium exclusion. Biochem. Bioph. Res. Co. 323, 534-540. doi: 10.1016/j.bbrc.2004.08.127

Supek, F., Bošnjak, M., Škunca, N, and Šmuc, T. (2011). REVIGO summarizes and visualizes long lists of gene ontology terms. PLoS ONE 6:e21800. doi: 10.1371/journal.pone.0021800

Vailleau, F., Daniel, X., Tronchet, M., Montillet, J. L., Triantaphylidès, C., and Roby, D. (2002). A R2R3-MYB gene, AtMYB30, acts as a positive regulator of the hypersensitive cell death program in plants in response to pathogen attack. Proc. Natl. Acad. Sci. U.S.A. 99, 10179-10184. doi: 10.1073/pnas.152047199

Ward, N., and Moreno-Hagelsieb, G. (2014). Quickly finding orthologs as reciprocal best hits with BLAT, LAST, and UBLAST: how much do we miss? PLoS ONE 9: e101850. doi: 10.1371/journal.pone.0101850

Yang, X., Deng, F., and Ramonell, K. M. (2012). Receptor-like kinases and receptor-like proteins: keys to pathogen recognition and defense signaling in plant innate immunity. Front Biol. 7, 155-166. doi: 10.1007/s11515-011-1 185-8

Yang, Y., Li, X., Yang, S., Zhou, Y., Dong, C., Ren, J., et al. (2015). Comparative physiological and proteomic analysis reveals the leaf response to cadmiuminduced stress in poplar (Populus yunnanensis). PLoS ONE 10:e0137396. doi: 10.1371/journal.pone.0137396

Yang, Z., and Bielawski, J. P. (2000). Statistical methods for detecting molecular adaptation. Trends Ecol Evol. 15, 496-503.

Zhan, Z., Wang, Y., Shockey, J., Chen, Y., Zhou, Z., Yao, X., et al. (2012). Breeding status of tung tree (Vernicia sp.) in China, a multipurpose oilseed crop with industrial uses. Silvae Genet. 61, 265-270

Zhang, W., Chen, J., Zhang, H., and Song, F. (2008). Overexpression of a rice diacylglycerol kinase gene OsBIDK1 enhances disease resistance in transgenic tobacco. Mol. Cells. 26, 258-264

Zhang, Y., Wang, X. F., Ding, Z. G., Ma, Q., Zhang, G. R., Zhang, S. L., et al. (2013). Transcriptome profiling of Gossypium barbadense inoculated with Verticillium dahliae provides a resource for cotton improvement. BMC Genomics 14:637. doi: 10.1186/1471-2164-14-637

Zhang, Z., Li, J., Zhao, X. Q., Wang, J., Wong, G. K., and Yu, J. (2006). KaKs Calculator: Calculating $\mathrm{Ka}$ and Ks through model selection and model averaging. Genomics Proteomics Bioinformatics 4, 259-263. doi: 10.1016/S1672-0229(07)60007-2

Zhang, Z., Liu, X., Wang, X., Zhou, M., Zhou, X., Ye, X., et al. (2012). An R2R3 MYB transcription factor in wheat, TaPIMP1, mediates host resistance to Bipolaris sorokiniana and drought stresses through regulation of defense- and stress-related genes. New Phytol. 196, 1155-1170. doi: $10.1111 /$ j.1469-8137.2012.04353.x

Conflict of Interest Statement: The authors declare that the research was conducted in the absence of any commercial or financial relationships that could be construed as a potential conflict of interest.

Copyright (c) 2016 Chen, Yin, Gao, Zhu, Zhang and Wang. This is an openaccess article distributed under the terms of the Creative Commons Attribution License (CC BY). The use, distribution or reproduction in other forums is permitted, provided the original author(s) or licensor are credited and that the original publication in this journal is cited, in accordance with accepted academic practice. No use, distribution or reproduction is permitted which does not comply with these terms. 